\title{
LESO Based Backstepping for Quadrotor Trace Tracking with Tracking Error Constraints
}

\author{
Fengjie $\mathrm{Gao}^{1, \mathrm{a}}$, Jing Xin ${ }^{2, \mathrm{~b}}$ and Rui Ding ${ }^{3, \mathrm{c}}$ \\ ${ }^{1}$ HIWING General Aviation Equipment Co.,Ltd., Beijing, China \\ ${ }^{2}$ HIWING General Aviation Equipment Co.,Ltd., Beijing, China \\ ${ }^{3}$ HIWING General Aviation Equipment Co.,Ltd., Beijing, China \\ ${ }^{a}$ Fengjie_gao@163.com, ${ }^{b}$ xinjing@hiwingage.com, ${ }^{c}$ dingrui@hiwingage.com
}

Keywords: trace tracking control; error constraints; LESO; backstepping; uncertainties

\begin{abstract}
The trace tracking control for quadrotor aircraft with system uncertainties and constraints for output tracking error is addressed. Uncertainties in quadrotor system is estimated by utilizing linear extended state observer. In addition, the backstepping method using barrier Lyapunov function is designed to compensate for quadrotor uncertainties while satisfying output tracking error constraints. In this way, the quadrotor tracking performance is improved. The closed-loop stability of the quadrotor under the proposed observer-controller structure is proven through Lyapunov theory. Efficiency of the proposed method is demonstrated with a quadrotor model subjected to parameter variation and external disturbance.
\end{abstract}

\section{Introduction}

Quadrotor aircraft has been used in military and civilian application owing to its aggressive maneuverability, simplicity of mechanics and increased payload [1]. However the trace tracking control of quadrotor aircraft is a challenging problem due to its nonlinear and coupling properties. Until now, a wide range of control methods have been proposed to address the quadrotor trace tracking problem. Linear control methods like LQR control, PID control are proposed in [2], nevertheless those methods highly depend on model accuracy, and linearization around the equilibrium point restricts the convergence region. Nonlinear methods such as sliding model control, model predictive control are presented in $[3,4]$, however the chattering output may make system unstable. Backstepping is one of the key methods to tackle quadrotor position control [5], but the weakness in disturbance suppression limits its application in real flight environment.

In real flight condition, the performance of quadrotor aircraft will be deteriorated by disturbance such as parameter uncertainties, wind drag force and load changes. To deal with system uncertainties, integral backstepping method was developed for quadrotor position control in [6]. Integral item helps to compensate system uncertainties, however it causes oscillation during dynamic response period. A nonlinear adaptive fuzzy control law was proposed in [1] to estimate quadrotor parameters to get an accurate model of quadrotor, however a lot of parameters have to be estimated which is quite time consuming.

The extended state observer (ESO) was first proposed by Han in 1990s [7]. The basic idea behind ESO is to view disturbance as an extended state and utilize observer to estimate it. With the development of ESO, some new forms like high order ESO, reduced ESO are proposed in [8,9]. Extensive researches are carried out to analyze the capability of ESO in the view of frequency domain and time domain $[8,10]$. ESO can largely be divided into two categories: linear extended state observer and nonlinear extended state observer. Due to the simplicity of tuning parameters, LESO has been widely used in practice.

Without prior knowledge of the desired trace, there is no way to find a control method for quadrotor trace tracking without any error. So it is meaningful to find a solution to keep the tracking error within a bound. To the best of our knowledge, there is no result on the problem of quadrotor trace tracking with output error constraints.

In order to deal with quadrotor trace tracking problem with output tracking error constraints, 
LESO based backstepping method using barrier Lyapunov function is proposed. The proposed method employs observer-controller structure. To simplify controller design, the quadrotor dynamic model is separated into underactuated subsystem and fully actuated subsystem referring to [4]. For underactuated subsystem, external disturbance and model uncertainties are estimated by two LESO. And for fully actuated subsystem, one LESO is designed. Backstepping method using barrier Lyapunov function is presented to compensate for quadrotor disturbance while satisfying output tracking error tolerance. Through this way, the output constraints are satisfied for quadrotor trace tracking in the present of disturbance.

\section{Quadrotor Modeling and Problem Formulation}

Quadrotor Dynamic Modeling. The quadrotor aircraft is shown in Fig. 1. In this paper, Euler angle roll $(\phi)$, pitch $(\theta)$, yaw $(\psi)$ are used to describe quadrotor attitude.
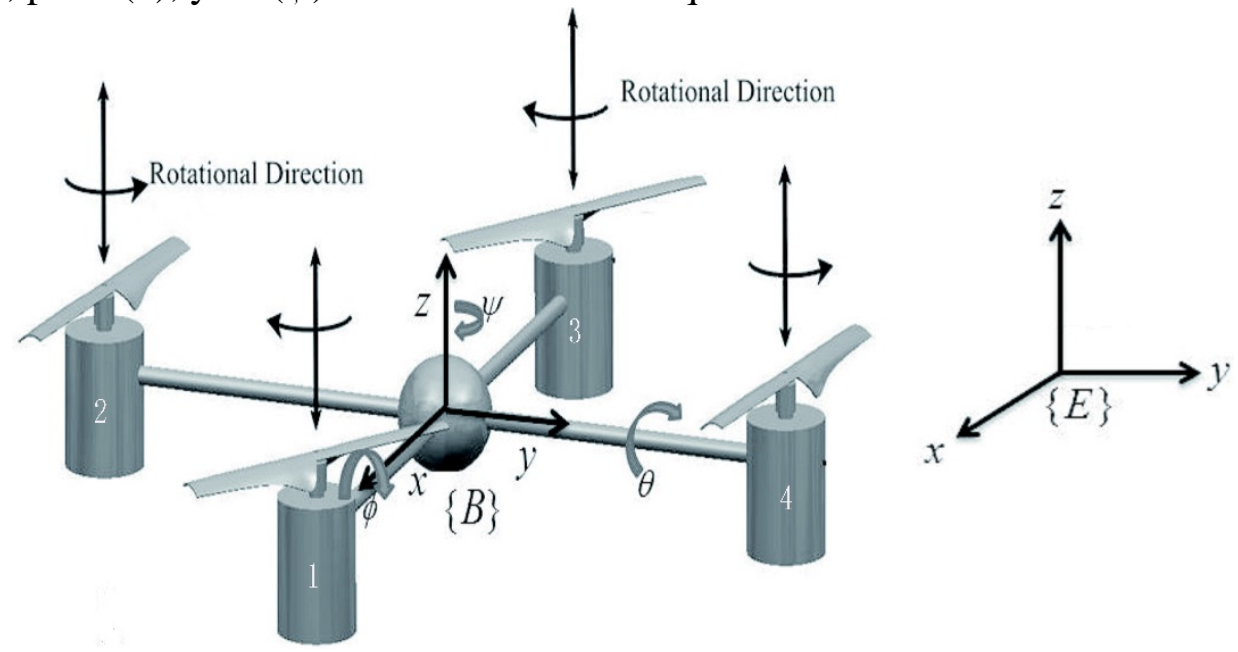

Fig. 1 Quadrotor Aircraft

In order to better describe the dynamic of quadrotor aircraft, body fixed frame $\{\mathrm{B}\}$ with origin located on quadrotor center and earth fixed frame $\{E\}$ is utilized. The quadrotor airframe in the space is given by a translational matrix $R_{t}$ from body fixed frame $\{\mathrm{B}\}$ to frame $\{\mathrm{E}\}$. Translational matrix $R_{t}$ is defined as Eq. 1.

$$
R_{t}=\left[\begin{array}{ccc}
C_{\phi} C_{\psi} & S_{\phi} S_{\theta} C_{\psi}-C_{\phi} S_{\psi} & C_{\phi} S_{\theta} C_{\psi}+S_{\phi} S_{\psi} \\
C_{\theta} S_{\psi} & S_{\phi} S_{\theta} S_{\psi}+C_{\phi} C_{\psi} & C_{\phi} S_{\theta} S_{\psi}-S_{\phi} C_{\psi} \\
-S_{\phi} & S_{\phi} C_{\theta} & C_{\phi} C_{\theta}
\end{array}\right]
$$

where $S_{(\cdot)}$ and $C_{(\cdot)}$ are abbreviations for $\sin (\cdot)$ and $\cos (\cdot)$ respectively.

The quadrotor can be viewed as a symmetric rigid body with six degrees of freedom. Let $\Sigma F$ denote sum of quadrotor propeller lift force and wind drag force. Let $\Sigma M$ denote sum of moments generated by propeller force and wind drag force. The dynamic motion equation of quadrotor in earth fixed frame $\{\mathrm{E}\}$ can be obtained through Newton-Euler principle and shown in Eq. 2.

$$
\left\{\begin{array}{l}
\Sigma F=m \ddot{\zeta} \\
\Sigma M=I_{b} \dot{\Omega}_{B}+\Omega_{B} \times I_{b} \Omega_{B}
\end{array}\right.
$$

where $\zeta \in \mathbb{R}^{3}$ denotes quadrotor location $x, y, z$ in the earth fixed frame $\{\mathrm{E}\}, \Omega_{B} \in \mathbb{R}^{3}$ is rotational angular velocity, mass of the quadrotor is represented by $m$, denotes inertia moments $I_{x x}, I_{y y}, I_{z z}$ about axis $x, y, z$ respectively.

Considering Eq. 1, Eq. 2 and transform the rotational motion into frame $\{\mathrm{E}\}$, the dynamic model of quadrotor in the earth fixed frame can be obtained [4]: 


$$
\left\{\begin{array}{llc}
\ddot{x} & = & \frac{1}{m}\left(C_{\phi} S_{\theta} C_{\psi}+S_{\phi} S_{\psi}\right) u_{1}-\frac{K_{1} \dot{x}}{m} \\
\ddot{y} & = & \frac{1}{m}\left(C_{\phi} S_{\theta} S_{\psi}-S_{\phi} C_{\psi}\right) u_{1}-\frac{K_{2} \dot{y}}{m} \\
\ddot{z} & = & \frac{1}{m}\left(C_{\phi} C_{\theta}\right) u_{1}-g-\frac{K_{3} \dot{z}}{m} \\
\ddot{\phi} & =\dot{\theta} \dot{\psi} \frac{I_{y y}-I_{z z}}{I_{x x}}+\frac{l}{I_{x x}} u_{2}-\frac{K_{4} l}{I_{x x}} \dot{\phi} \\
\ddot{\theta} & =\dot{\psi} \dot{\phi} \frac{I_{z z}-I_{x x}}{I_{y y}}+\frac{l}{I_{y y}} u_{3}-\frac{K_{5} l}{I_{y y}} \dot{\theta} \\
\ddot{\psi} & = & \dot{\phi} \dot{\theta} \frac{I_{x x}-I_{y y}}{I_{z z}}+\frac{1}{I_{z z}} u_{4}-\frac{K_{6} l}{I_{z z}} \dot{\psi}
\end{array}\right.
$$

where $K_{i}(i=1,2,3,4,5,6)$ denotes the wind drag force coefficient, $l$ is lever length.

Problem Formulation. The control input of quadrotor is $u_{1}, u_{2}, u_{3}, u_{4}$, the output is its location $(x, y, z)$ and Euler angle $(\phi, \theta, \psi)$. Quadrotor has six outputs while it only has four inputs, thus the quadrotor is an under actuated system. In the task of trace tracking, position $x, y, z$ must be chosen as the controller output. And yaw angle $\psi$ is selected to be the only controlled angle, because tilt angle $\phi$ and $\theta$ are always small in the process of steady flying while the yaw angle $\psi$ adjustment is normally crucial in practice. So the objective of controller design is to ensure the output $x(t), y(t), z(t), \psi(t)$ to follow desired trace $x_{d}(t), y_{d}(t), z_{d}(t), \psi_{d}(t)$ in the presence of uncertainties. In order to derive backstepping control law for quadrotor trace tracking, quadrotor dynamic model as Eq. 3 is divided into subsystem $S_{1}$ and $S_{2}$ as Eq. 4.

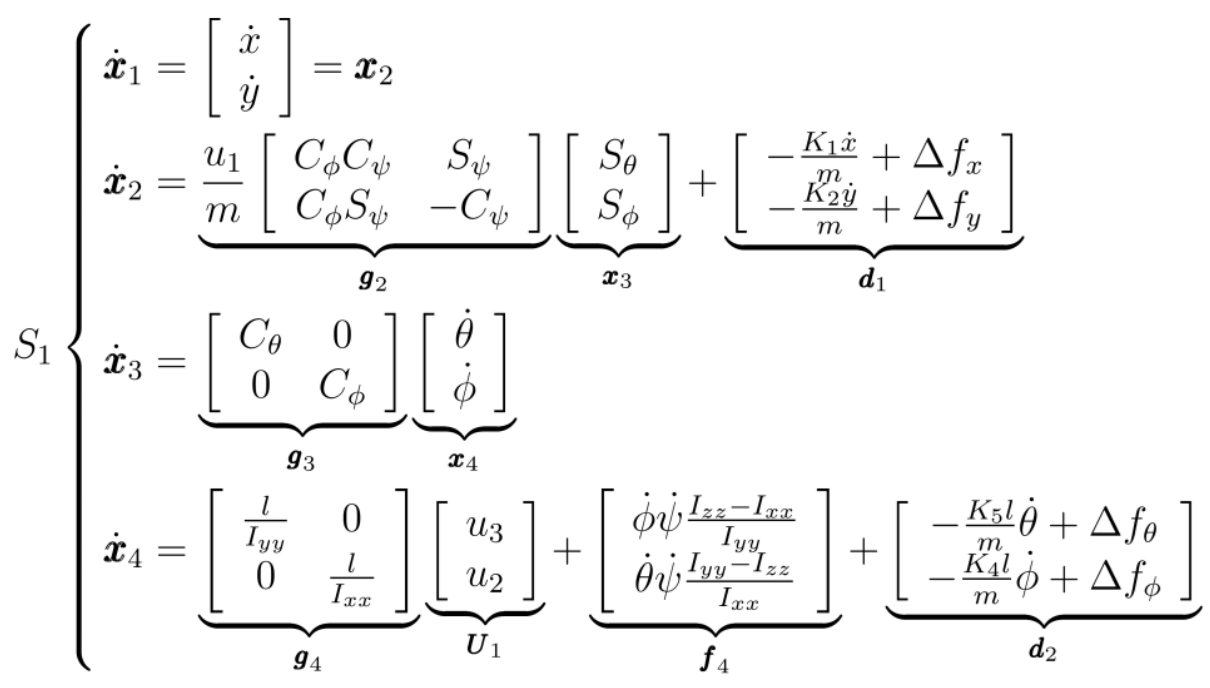

$$
\begin{aligned}
& S_{2}\left\{\begin{array}{l}
\dot{\boldsymbol{x}}_{5}=\left[\begin{array}{c}
\dot{z} \\
\dot{\psi}
\end{array}\right]=\boldsymbol{x}_{6} \\
\dot{\boldsymbol{x}}_{6}=\underbrace{\left[\begin{array}{cc}
\frac{C_{\phi} C_{\theta}}{m} & 0 \\
0 & \frac{l}{I_{z z}}
\end{array}\right]}_{\boldsymbol{g}_{6}} \underbrace{\left[\begin{array}{c}
u_{1} \\
u_{4}
\end{array}\right]}_{\boldsymbol{U}_{2}}+\underbrace{\left[\begin{array}{c}
-g \\
0
\end{array}\right]}_{\boldsymbol{f}_{6}}+\underbrace{\left[\begin{array}{c}
-\frac{K_{3} \dot{z}}{m}+\Delta f_{z} \\
-\frac{K_{6} l}{m} \dot{\phi}+\Delta f_{\psi}
\end{array}\right]}_{\boldsymbol{d}_{3}}
\end{array}\right.
\end{aligned}
$$

where $\Delta f_{(\cdot)}$ are model uncertainties consisting of parameter variation and external disturbance. Since the wind drag forces modeled in Eq. 3 are small and not easy to be measured, they are regarded as external disturbance in the controller design procedure. That is to say, the total disturbance $\boldsymbol{d}_{i}(i=1,2,3)$ consists of model uncertainties $\Delta f_{(\cdot)}$ and wind drag force modeled in Eq. 3.

\section{LESO and Controller Design}

Linear Extended State Observer Design. In this section, linear extended state observers are designed to estimate disturbances $\boldsymbol{d}_{1}, \boldsymbol{d}_{2}$ in the under actuated subsystem $S_{1}$ and disturbance $\boldsymbol{d}_{3}$ in the fully actuated subsystem $S_{2}$. In the design procedure, we assume that all states are measurable. The observers are described by Eq. 5 . 


$$
\left\{\begin{array}{l}
\dot{z}_{2}=g_{2} x_{3}+z_{3}+2 \omega_{1}\left(x_{2}-z_{2}\right) \\
\dot{z}_{3}=\omega_{1}^{2}\left(x_{2}-z_{2}\right) \\
\dot{z}_{4}=g_{4} U_{1}+f_{4}+z_{5}+2 \omega_{2}\left(x_{4}-z_{4}\right) \\
\dot{z}_{5}=\omega_{2}^{2}\left(x_{4}-z_{4}\right) \\
\dot{z}_{6}=g_{6} U_{2}+f_{6}+z_{7}+2 \omega_{3}\left(x_{6}-z_{6}\right) \\
\dot{z}_{7}=\omega_{3}^{2}\left(x_{6}-z_{6}\right)
\end{array}\right.
$$

where $\boldsymbol{z}_{i}(i=2,3,4,5,6,7)$ are the states of LESO, $\boldsymbol{\omega}_{i}(i=1,2,3) \in \mathbb{R}^{2 \times 2}$ are positive definite diagonal matrixes, and $z_{3}, z_{5}, z_{7}$ are the estimation of total disturbance $\boldsymbol{d}_{1}, \boldsymbol{d}_{2}, \boldsymbol{d}_{3}$ respectively. For convenience of expression, let $\hat{d}_{1}=z_{3}, \hat{d}_{2}=z_{5}, \hat{d}_{3}=z_{7}$. It should be noted that for the designed LESO, only three diagonal matrixes $\boldsymbol{w}_{1}, \boldsymbol{w}_{2}, \boldsymbol{w}_{3}$ need to be tuned.

Assumption 1: The disturbance $\boldsymbol{d}_{1}, \boldsymbol{d}_{2}, \boldsymbol{d}_{3}$ are bounded, and their derivatives are also bounded, such that $\left|\dot{\boldsymbol{d}}_{1}\right| \leqslant \boldsymbol{l}_{1},\left|\dot{\boldsymbol{d}}_{2}\right| \leqslant \boldsymbol{l}_{2},\left|\dot{\boldsymbol{d}}_{3}\right| \leqslant \boldsymbol{l}_{3}$, where $\boldsymbol{l}_{1}, \boldsymbol{l}_{2}, \boldsymbol{l}_{3} \in \mathbb{R}^{2 \times 1}$ are constant vectors.

Let $E_{1}=\left[\boldsymbol{x}_{2}-\boldsymbol{z}_{2} ; \boldsymbol{d}_{1}-\hat{\boldsymbol{d}}_{1}\right], E_{2}=\left[\boldsymbol{x}_{4}-\boldsymbol{z}_{4} ; \boldsymbol{d}_{2}-\hat{\boldsymbol{d}}_{2}\right], E_{3}=\left[\boldsymbol{x}_{6}-\boldsymbol{z}_{6} ; \boldsymbol{d}_{3}-\hat{\boldsymbol{d}}_{3}\right] . E_{1}, E_{2}, E_{3} \in \mathbb{R}^{4 \times 1}$. Then the observer errors can be obtain via Eq. 6 [10].

$$
\dot{E}_{i}=A_{i} E_{i}+B \dot{\boldsymbol{d}}_{i} \quad(i=1,2,3)
$$

where $A_{i}$ is Hurwitz matrix. $A_{i}, B$ are defined as:

$$
A_{i}=\left[\begin{array}{cc}
-2 \boldsymbol{w}_{i} & I_{2 \times 2} \\
-\boldsymbol{w}_{i}^{2} & \mathbf{0}
\end{array}\right], B=\left[\begin{array}{c}
\mathbf{0} \\
I_{2 \times 2}
\end{array}\right]
$$

Let $E_{i}=\left[\widetilde{\boldsymbol{x}}_{2 i}, \widetilde{\boldsymbol{d}}_{i}\right]^{T}$, then one can get the time derivative of $\widetilde{\boldsymbol{d}}_{i}$ as:

$$
\dot{\tilde{d}}_{i}=-\boldsymbol{w}_{i}^{2} \widetilde{\boldsymbol{x}}_{2 i}+\dot{\boldsymbol{d}}_{i}
$$

Solving Eq. 6, one can obtain:

$$
\left|E_{i}(t)\right|=e^{A_{i} t}\left|E_{i}(0)\right|+\left|A_{i}^{-1}\left(I_{4 \times 4}-e^{A_{i} t}\right) B\right| \boldsymbol{l}_{i}
$$

Therefore $\left|E_{i}(t)\right| \leqslant e^{A_{i} t}\left|E_{i}(0)\right|+\left|A_{i}^{-1} B\right| \boldsymbol{l}_{i}$. The disturbance observer errors $\widetilde{\boldsymbol{d}}_{1}, \widetilde{\boldsymbol{d}}_{2}, \widetilde{\boldsymbol{d}}_{3}$ are bounded.

Controller Design. The underactuated subsystem $S_{1}$ is controlled to drive the quadrotor output $x(t), y(t)$ to follow the desired values $x_{d}(t), y_{d}(t)$ and the fully subsystem $S_{2}$ is controlled to drive the quadrotor output $z(t), \psi(t)$ to follow the desired values $z_{d}(t), \psi_{d}(t)$. Further more, the trace tracking error will be bounded in the presence of the total disturbance. The controller design procedure for underactuated subsystem is shown from step 1 to step 4, step 5 shows the fully subsystem controller design procedure.

Step 1: In the first step, we construct the virtual system

$$
\dot{x}_{1}=v_{1}
$$

Let $\xi_{1}$ denote the subsystem trace tracking error, then

$$
\xi_{1}=x_{1 d}-x_{1}
$$

Setting error tolerance for underactuated subsystem to be $k_{b 1}$ and constructing a positive 
defined Lyapunov function

$$
V_{1}=\frac{1}{2} \log \left(\frac{\boldsymbol{k}_{b 1}^{T} \boldsymbol{k}_{b 1}}{\boldsymbol{k}_{b 1}^{T} \boldsymbol{k}_{b 1}-\boldsymbol{\xi}_{1}^{T} \boldsymbol{\xi}_{1}}\right)
$$

In the virtual system, the time derivative of Eq. 12 is :

$$
\dot{V}_{1}=\frac{\boldsymbol{\xi}_{1}^{T} \dot{\boldsymbol{\xi}}_{1}}{\boldsymbol{k}_{b 1}^{T} \boldsymbol{k}_{b 1}-\boldsymbol{\xi}_{1}^{T} \xi_{1}}=\frac{\boldsymbol{\xi}_{1}^{T}\left(\dot{\boldsymbol{x}}_{1 d}-\boldsymbol{v}_{1}\right)}{\boldsymbol{k}_{b 1}^{T} \boldsymbol{k}_{b 1}-\boldsymbol{\xi}_{1}^{T} \boldsymbol{\xi}_{1}}
$$

The subsystem trace tracking error $\xi_{1}$ can be stabilized by setting the first virtual input $\boldsymbol{v}_{1}$ as:

$$
\boldsymbol{v}_{1}=\left(\boldsymbol{k}_{b 1}^{T} \boldsymbol{k}_{b 1}-\boldsymbol{\xi}_{1}^{T} \boldsymbol{\xi}_{1}\right) A_{1} \boldsymbol{\xi}_{1}+\dot{\boldsymbol{x}}_{1 d}
$$

where $A_{1} \in \mathbb{R}^{2 \times 2}$ is a positive definite diagonal matrix.

Substituting Eq. 14 into Eq. 13, one can get:

$\dot{V}_{1}=-\xi_{1}^{T} A_{1} \xi_{1}<0$

Step 2: In this step, we construct the second virtual system as:

$\dot{x}_{2}=\boldsymbol{g}_{2} \boldsymbol{v}_{2}+\boldsymbol{d}_{1}$

Let $\xi_{2}$ denote the virtual control error of $\boldsymbol{v}_{1}$ :

$\boldsymbol{\xi}_{2}=\boldsymbol{v}_{1}-\boldsymbol{x}_{2}=\left(\boldsymbol{k}_{b 1}^{T} \boldsymbol{k}_{b 1}-\boldsymbol{\xi}_{1}^{T} \boldsymbol{\xi}_{1}\right) A_{1} \boldsymbol{\xi}_{1}+\dot{\boldsymbol{x}}_{1 d}-\boldsymbol{x}_{2}$

In the second step, we construct the augmented Lyapunov function as:

$V_{2}=V_{1}+\frac{1}{2} \boldsymbol{\xi}_{2}^{T} \boldsymbol{\xi}_{2}+\frac{1}{2} \widetilde{\boldsymbol{d}}_{1}^{T} \widetilde{\boldsymbol{d}}_{1}$

The time derivative of the augmented Lyapunov function is

$\dot{V}_{2}=-\boldsymbol{\xi}_{1}^{T} A_{1} \xi_{1}+\boldsymbol{\xi}_{2}^{T}\left(\dot{\boldsymbol{v}}_{1}-\boldsymbol{g}_{2} \boldsymbol{v}_{2}-\hat{\boldsymbol{d}}_{1}+\frac{\boldsymbol{\xi}_{1}}{\left(\boldsymbol{k}_{b 1}^{T} \boldsymbol{k}_{b 1}-\boldsymbol{\xi}_{1}^{T} \boldsymbol{\xi}_{1}\right)}\right)-\boldsymbol{\xi}_{2}^{T} \widetilde{\boldsymbol{d}}_{1}+\widetilde{\boldsymbol{d}}_{1}^{T} \dot{\widetilde{\boldsymbol{d}}}_{1}$

Introducing the second virtual control $\boldsymbol{v}_{2}$ as Eq. 20,

$$
\boldsymbol{v}_{2}=\boldsymbol{g}_{2}^{-1}\left(\dot{\boldsymbol{v}}_{1}-\hat{\boldsymbol{d}}_{1}+\frac{\boldsymbol{\xi}_{1}}{\boldsymbol{k}_{b 1}^{T} \boldsymbol{k}_{b 1}-\boldsymbol{\xi}_{1}^{T} \boldsymbol{\xi}_{1}}+A_{2} \xi_{2}\right)
$$

where $A_{2} \in \mathbb{R}^{2 \times 2}$ is a positive definite diagonal matrix.

Remark 1: In the process of quadrotor flying, we have $\phi \in\left(-\frac{\pi}{2}, \frac{\pi}{2}\right)$ and $C_{\phi}>0$. Therefore matrix $\boldsymbol{g}_{2}$ is nonsingular since the determinant of matrix $\boldsymbol{g}_{2}$ is equal to $\left(-C_{\phi}\right)$. Substituting Eq. 8, Eq.20 into Eq. 19, one gets Eq. 21, where $M_{1}=\omega_{1}^{2}-\frac{1}{4} A_{2}^{-1}$. 


$$
\begin{aligned}
\dot{V}_{2}= & -\boldsymbol{\xi}_{1}^{T} A_{1} \boldsymbol{\xi}_{1}-\left\|A_{2}^{\frac{1}{2}} \boldsymbol{\xi}_{2}+\frac{1}{2} A_{2}^{-\frac{1}{2}} \widetilde{\boldsymbol{d}}_{1}\right\|_{2}^{2}-\left\|M_{1}^{\frac{1}{2}} \widetilde{\boldsymbol{d}}_{1}-\frac{1}{2} M_{1}^{-\frac{1}{2}}\left(\boldsymbol{\omega}_{1}^{2}\left(\widetilde{\boldsymbol{d}}_{1}-\widetilde{\boldsymbol{x}}_{2}\right)+\dot{\boldsymbol{d}}_{1}\right)\right\|_{2}^{2} \\
& +\left\|\frac{1}{2} M_{1}^{-\frac{1}{2}}\left(\boldsymbol{\omega}_{1}^{2}\left(\widetilde{\boldsymbol{d}}_{1}-\widetilde{\boldsymbol{x}}_{2}\right)+\dot{\boldsymbol{d}}_{1}\right)\right\|_{2}^{2}
\end{aligned}
$$

Remark 2: The elements of $A_{2}$ are chosen to satisfy $A_{2}^{-1}<4 w_{1}^{2}$, then $M_{1}$ is a positive definite matrix.

Step 3: In this step, we construct the third virtual system

$\dot{x}_{3}=g_{3} v_{3}$

Let $\xi_{3}$ denote the virtual control error of $\boldsymbol{v}_{2}$ :

$\boldsymbol{\xi}_{3}=\boldsymbol{v}_{2}-\boldsymbol{x}_{3}=\boldsymbol{g}_{2}^{-1}\left[\dot{\boldsymbol{v}}_{1}-\hat{\boldsymbol{d}}_{1}+\frac{\boldsymbol{\xi}_{1}}{\boldsymbol{k}_{b 1}^{T} \boldsymbol{k}_{b 1}-\boldsymbol{\xi}_{1}^{T} \boldsymbol{\xi}_{1}}+A_{2} \xi_{2}\right]-\boldsymbol{x}_{3}$

Then reshaping Eq. 23 as:

$\boldsymbol{g}_{2} \xi_{3}=\dot{\boldsymbol{v}}_{1}-\boldsymbol{g}_{2} \boldsymbol{x}_{3}-\boldsymbol{d}_{1}+\widetilde{\boldsymbol{d}}_{1}+\frac{\xi_{1}}{\boldsymbol{k}_{b 1}^{T} \boldsymbol{k}_{b 1}-\boldsymbol{\xi}_{1}^{T} \xi_{1}}+A_{2} \xi_{2}$

Considering the Lyapunov function $V_{3}$ :

$V_{3}=V_{2}+\frac{1}{2} \xi_{3}^{T} \xi_{3}$

The time derivative of Eq.25 can be obtained as the following equation:

$$
\begin{aligned}
\dot{V}_{3}= & \frac{\boldsymbol{\xi}_{1}^{T}\left[\boldsymbol{\xi}_{2}-\left(\boldsymbol{k}_{b 1}^{T} \boldsymbol{k}_{b 1}-\boldsymbol{\xi}_{1}^{T} \boldsymbol{\xi}_{1}\right) A_{1} \boldsymbol{\xi}_{1}\right]}{\boldsymbol{k}_{b 1}^{T} \boldsymbol{k}_{b 1}-\boldsymbol{\xi}_{1}^{T} \boldsymbol{\xi}_{1}}+\boldsymbol{\xi}_{2}^{T}\left[-\widetilde{\boldsymbol{d}}_{1}-\frac{\boldsymbol{\xi}_{1}}{\boldsymbol{k}_{b 1}^{T} \boldsymbol{k}_{b 1}-\boldsymbol{\xi}_{1}^{T} \boldsymbol{\xi}_{1}}-A_{2} \boldsymbol{\xi}_{2}\right]+\widetilde{\boldsymbol{d}}_{1}^{T} \dot{\boldsymbol{d}}_{1} \\
& +\boldsymbol{\xi}_{3}^{T}\left(\dot{\boldsymbol{v}}_{2}-\boldsymbol{g}_{3} \boldsymbol{v}_{3}+\boldsymbol{g}_{2} \boldsymbol{\xi}_{2}\right)
\end{aligned}
$$

Setting the third virtual control $\boldsymbol{v}_{3}$ as:

$$
\boldsymbol{v}_{3}=\boldsymbol{g}_{3}^{-1}\left[\dot{\boldsymbol{v}}_{2}+\boldsymbol{g}_{2} \xi_{2}+A_{3} \boldsymbol{\xi}_{3}\right]
$$

where $A_{3} \in \mathbb{R}^{2 \times 2}$ is a positive definite diagonal matrix.

Remark 3: In steady trace tracking course, tilt angle $\phi \in\left(-\frac{\pi}{2}, \frac{\pi}{2}\right), \theta \in\left(-\frac{\pi}{2}, \frac{\pi}{2}\right)$. Therefore matrix $\boldsymbol{g}_{3}$ is nonsingular since the determinant of matrix $\boldsymbol{g}_{3}$ is equal to $C_{\phi} C_{\theta}$.

Substituting Eq. 27 into Eq. 26, one gets

$$
\begin{aligned}
\dot{V}_{3}= & -\boldsymbol{\xi}_{1}^{T} A_{1} \boldsymbol{\xi}_{1}-\boldsymbol{\xi}_{3}^{T} A_{3} \boldsymbol{\xi}_{3}-\left\|A_{2}^{\frac{1}{2}} \boldsymbol{\xi}_{2}+\frac{1}{2} A_{2}^{-\frac{1}{2}} \widetilde{\boldsymbol{d}}_{1}\right\|_{2}^{2}-\left\|M_{1}^{\frac{1}{2}} \widetilde{\boldsymbol{d}}_{1}-\frac{1}{2} M_{1}^{-\frac{1}{2}}\left(\boldsymbol{\omega}_{1}^{2}\left(\widetilde{\boldsymbol{d}}_{1}-\widetilde{\boldsymbol{x}}_{2}\right)+\dot{\boldsymbol{d}}_{1}\right)\right\|_{2}^{2} \\
& +\left\|\frac{1}{2} M_{1}^{-\frac{1}{2}}\left(\boldsymbol{\omega}_{1}^{2}\left(\widetilde{\boldsymbol{d}}_{1}-\widetilde{\boldsymbol{x}}_{2}\right)+\dot{\boldsymbol{d}}_{1}\right)\right\|_{2}^{2}
\end{aligned}
$$

Step 4: In this step, considering the dynamic of $x_{4}$

$$
\dot{x}_{4}=g_{4} U_{1}+f_{4}+d_{2}
$$


Let

$\xi_{4}=\boldsymbol{v}_{3}-\boldsymbol{x}_{4}=\boldsymbol{g}_{3}^{-1}\left[\dot{\boldsymbol{v}}_{2}+\boldsymbol{g}_{2} \boldsymbol{\xi}_{2}+A_{3} \xi_{3}\right]-\boldsymbol{x}_{4}$

Then

$\boldsymbol{g}_{3} \xi_{4}=\dot{\boldsymbol{v}}_{2}-\boldsymbol{g}_{3} \boldsymbol{x}_{4}+\boldsymbol{g}_{2} \xi_{2}+A_{3} \boldsymbol{\xi}_{3}$

Setting the global Lyapunov function for the underactuated subsystem as:

$V_{4}=V_{3}+\frac{1}{2} \boldsymbol{\xi}_{4}^{T} \boldsymbol{\xi}_{4}+\frac{1}{2} \widetilde{\boldsymbol{d}}_{2}^{T} \widetilde{\boldsymbol{d}}_{2}$

The time derivative of $V_{4}$ is

$$
\begin{aligned}
\dot{V}_{4} & =\frac{\boldsymbol{\xi}_{1}^{T}\left[\boldsymbol{\xi}_{2}-\left(\boldsymbol{k}_{b 1}^{T} \boldsymbol{k}_{b 1}-\boldsymbol{\xi}_{1}^{T} \boldsymbol{\xi}_{1}\right) A_{1} \xi_{1}\right]}{\boldsymbol{k}_{b 1}^{T} \boldsymbol{k}_{b 1}-\boldsymbol{\xi}_{1}^{T} \boldsymbol{\xi}_{1}}+\widetilde{\boldsymbol{d}}_{1}^{T} \dot{\widetilde{\boldsymbol{d}}}_{1}+\boldsymbol{\xi}_{2}^{T}\left[\boldsymbol{g}_{2} \xi_{3}-\widetilde{\boldsymbol{d}}_{1}-\frac{\xi_{1}}{\boldsymbol{k}_{b 1}^{T} \boldsymbol{k}_{b 1}-\boldsymbol{\xi}_{1}^{T} \boldsymbol{\xi}_{1}}-A_{2} \boldsymbol{\xi}_{2}\right] \\
& +\widetilde{\boldsymbol{d}}_{2}^{T} \dot{\tilde{\boldsymbol{d}}}_{2}-\boldsymbol{\xi}_{3}^{T}\left(\boldsymbol{g}_{2} \boldsymbol{\xi}_{2}+A_{3} \boldsymbol{\xi}_{3}\right)+\boldsymbol{\xi}_{4}^{T}\left(\boldsymbol{g}_{3} \boldsymbol{\xi}_{3}+\dot{\boldsymbol{v}}_{3}-\boldsymbol{g}_{4} \boldsymbol{U}_{1}-\boldsymbol{f}_{4}-\boldsymbol{d}_{2}\right)
\end{aligned}
$$

Setting the control law of underactuated subsystem as

$$
U_{1}=g_{4}^{-1}\left(\dot{v}_{3}-f_{4}-\hat{d}_{2}+g_{3} \xi_{3}+A_{4} \xi_{4}\right)
$$

Then similar to the reshaping of $\dot{V}_{2}$, one can substitute Eq.34 into Eq. 33 and get the derivative of $V_{4}$ as Eq. 35, where $M_{2}=\omega_{2}^{2}-\frac{1}{4} A_{4}^{-1}$.

$$
\begin{aligned}
\dot{V}_{4}= & -\boldsymbol{\xi}_{1}^{T} A_{1} \boldsymbol{\xi}_{1}-\boldsymbol{\xi}_{3}^{T} A_{3} \boldsymbol{\xi}_{3}-\left\|A_{2}^{\frac{1}{2}} \boldsymbol{\xi}_{2}+\frac{1}{2} A_{2}^{-\frac{1}{2}} \widetilde{\boldsymbol{d}}_{1}\right\|_{2}^{2}-\left\|M_{1}^{\frac{1}{2}} \widetilde{\boldsymbol{d}}_{1}-\frac{1}{2} M_{1}^{-\frac{1}{2}}\left(\boldsymbol{\omega}_{1}^{2}\left(\widetilde{\boldsymbol{d}}_{1}-\widetilde{\boldsymbol{x}}_{2}\right)+\dot{\boldsymbol{d}}_{1}\right)\right\|_{2}^{2} \\
& +\left\|\frac{1}{2} M_{1}^{-\frac{1}{2}}\left(\boldsymbol{\omega}_{1}^{2}\left(\widetilde{\boldsymbol{d}}_{1}-\widetilde{\boldsymbol{x}}_{2}\right)+\dot{\boldsymbol{d}}_{1}\right)\right\|_{2}^{2}-\left\|A_{4}^{\frac{1}{2}} \boldsymbol{\xi}_{4}+\frac{1}{2} A_{4}^{-\frac{1}{2}} \widetilde{\boldsymbol{d}}_{2}\right\|_{2}^{2} \\
& -\left\|M_{2}^{\frac{1}{2}} \widetilde{\boldsymbol{d}}_{2}-\frac{1}{2} M_{2}^{-\frac{1}{2}}\left(\boldsymbol{\omega}_{2}^{2}\left(\widetilde{\boldsymbol{d}}_{2}-\widetilde{\boldsymbol{x}}_{4}\right)+\dot{\boldsymbol{d}}_{2}\right)\right\|_{2}^{2}+\left\|\frac{1}{2} M_{2}^{-\frac{1}{2}}\left(\boldsymbol{\omega}_{2}^{2}\left(\widetilde{\boldsymbol{d}}_{2}-\widetilde{\boldsymbol{x}}_{4}\right)+\dot{\boldsymbol{d}}_{2}\right)\right\|_{2}^{2}
\end{aligned}
$$

Remark 4: The elements of $A_{4}$ are chosen to satisfy $A_{4}^{-1}<4 \omega_{2}^{2}$, then $M_{2}$ is a positive definite matrix.

Remark 5: From Eq. 9, we can get $\widetilde{\boldsymbol{x}}_{2 i}, \widetilde{\boldsymbol{d}}_{i}$ are bounded. So $\left\|\frac{1}{2} M_{i}^{-\frac{1}{2}}\left(\boldsymbol{\omega}_{i}^{2}\left(\widetilde{\boldsymbol{d}}_{i}-\widetilde{\boldsymbol{x}}_{2 i}\right)+\dot{\boldsymbol{d}}_{i}\right)\right\|_{2}^{2}$ $(i=1,2)$ is bounded. Thus the trace tracking error $\xi_{1}$ of underactuated subsystem will satisfy the constraint $\left|\xi_{1}\right|<\boldsymbol{k}_{b 1}$, and all states of underactuated subsystem $X_{u}(t)$ will stay within the boundary $B_{u}$ where $\left.B_{u}=\sum_{i=1}^{2}\left(\left\|\frac{1}{2}\left(\boldsymbol{\omega}_{i}^{2}-\frac{1}{4} A_{2 i}^{-1}\right)^{-\frac{1}{2}}\left(\boldsymbol{\omega}_{i}^{2}\left(\widetilde{\boldsymbol{d}}_{i}-\widetilde{\boldsymbol{x}}_{2 i}\right)+\dot{\boldsymbol{d}}_{i}\right)\right\|_{2}^{2}\right)\right\}$.

Step 5: Since the procedure of synthesizing control law for fully actuated subsystem $S_{2}$ is similar to that of underactuated subsystem, we just give the control law and time derivative of global Lyapunov function for $S_{2}$ in the fifth step. The error tolerance for $S_{2}$ is set to be $k_{b 2}$. The control law for $S_{2}$ is as Eq. 36, where $A_{5}, A_{6} \in \mathbb{R}^{2 \times 2}$ are positive definite matrixes. The elements of $A_{6}$ are chosen to satisfy $A_{6}^{-1}<4 \omega_{3}^{2}$. 


$$
\left\{\begin{aligned}
\boldsymbol{\xi}_{5} & =\boldsymbol{x}_{5 d}-\boldsymbol{x}_{5} \\
\boldsymbol{v}_{5} & =\left(\boldsymbol{k}_{b 2}^{T} \boldsymbol{k}_{b 2}-\boldsymbol{\xi}_{5}^{T} \boldsymbol{\xi}_{5}\right) A_{5} \boldsymbol{\xi}_{5}+\dot{\boldsymbol{x}}_{5 d} \\
\boldsymbol{\xi}_{6} & =\boldsymbol{v}_{5}-\boldsymbol{x}_{6} \\
\boldsymbol{U}_{2} & =\boldsymbol{g}_{6}^{-1}\left[\dot{\boldsymbol{v}}_{5}-\boldsymbol{f}_{6}-\hat{\boldsymbol{d}}_{3}+\frac{\boldsymbol{\xi}_{5}}{\left(\boldsymbol{k}_{b 2}^{T} \boldsymbol{k}_{b 2}-\boldsymbol{\xi}_{5}^{T} \boldsymbol{\xi}_{5}\right)}+A_{6} \boldsymbol{\xi}_{6}\right]
\end{aligned}\right.
$$

In this step, the barrier Lyapunov function is defined as:

$$
V_{5}=\frac{1}{2} \log \frac{\boldsymbol{k}_{b 2}^{T} \boldsymbol{k}_{b 2}}{\boldsymbol{k}_{b 2}^{T} \boldsymbol{k}_{b 2}-\boldsymbol{\xi}_{5}^{T} \boldsymbol{\xi}_{5}}
$$

The global Lyapunov function for fully actuated subsystem is defined as:

$$
V_{6}=V_{5}+\frac{1}{2} \boldsymbol{\xi}_{6}^{T} \boldsymbol{\xi}_{6}+\frac{1}{2} \widetilde{\boldsymbol{d}}_{3}^{T} \widetilde{\boldsymbol{d}}_{3}
$$

Considering Eq.36, Eq.37, Eq.38, one can get the time derivative of $V_{6}$ as:

$$
\begin{aligned}
\dot{V}_{6}= & \dot{V}_{5}+\boldsymbol{\xi}_{6}^{T} \dot{\boldsymbol{\xi}}_{6}+\widetilde{\boldsymbol{d}}_{3}^{T} \dot{\overrightarrow{\boldsymbol{d}}}_{3} \\
= & -\boldsymbol{\xi}_{5}^{T} A_{5} \boldsymbol{\xi}_{5}-\left\|A_{6}^{\frac{1}{2}} \boldsymbol{\xi}_{6}+\frac{1}{2} A_{6}^{-\frac{1}{2}} \widetilde{\boldsymbol{d}}_{3}\right\|_{2}^{2}-\left\|M_{3}^{\frac{1}{2}} \widetilde{\boldsymbol{d}}_{3}-\frac{1}{2} M_{3}^{-\frac{1}{2}}\left(\boldsymbol{\omega}_{3}^{2}\left(\widetilde{\boldsymbol{d}}_{3}-\widetilde{\boldsymbol{x}}_{6}\right)+\dot{\boldsymbol{d}}_{3}\right)\right\|_{2}^{2} \\
& +\left\|\frac{1}{2} M_{3}^{-\frac{1}{2}}\left(\boldsymbol{\omega}_{3}^{2}\left(\widetilde{\boldsymbol{d}}_{3}-\widetilde{\boldsymbol{x}}_{6}\right)+\dot{\boldsymbol{d}}_{3}\right)\right\|_{2}^{2}
\end{aligned}
$$

where $M_{3}=\omega_{3}^{2}-\frac{1}{4} A_{6}^{-1}$.

Remark 6: From Eq. 9, we can get $\widetilde{\boldsymbol{x}}_{5}, \widetilde{\boldsymbol{d}}_{3}$ are bounded. Thus the trace tracking error $\xi_{5}$ of fully actuated subsystem will satisfy the constraint $\left|\boldsymbol{\xi}_{5}\right|<\boldsymbol{k}_{b 2}$, and all states of fully actuated subsystem $X_{f}(t)$ will stay within boundary $B_{f}$ where $\left.B_{f}=\left\|\frac{1}{2}\left(\boldsymbol{\omega}_{3}^{2}-\frac{1}{4} A_{6}^{-1}\right)^{-\frac{1}{2}}\left(\boldsymbol{\omega}_{3}^{2}\left(\widetilde{\boldsymbol{d}}_{3}-\widetilde{\boldsymbol{x}}_{6}\right)+\dot{\boldsymbol{d}}_{3}\right)\right\|_{2}^{2}\right\}$.

\section{Simulation Result}

\begin{tabular}{|c|c|c|c|c|c|c|c|}
\hline \multicolumn{6}{|c|}{ Q Quadrotor Dynamic } & LESO Parameters & Controller Parameters \\
\hline$m$ & 1.2 & {$[k g]$} & $K_{1}$ & 0.5 & {$[\mathrm{~kg} / \mathrm{s}]$} & $\boldsymbol{\omega}_{1} \quad \operatorname{diag}[10,10]$ & $\operatorname{diag}[10,10]$ \\
\hline$g$ & 9.81 & {$\left[\mathrm{~m} / \mathrm{s}^{2}\right]$} & $K_{2}$ & 0.5 & {$[\mathrm{~kg} / \mathrm{s}]$} & $\boldsymbol{\omega}_{3} \operatorname{diag}[10,10]$ & $A_{4} \quad \operatorname{diag}[0.08,0.08]$ \\
\hline$l$ & 0.5 & {$[m]$} & $K_{3}$ & 0.5 & {$[\mathrm{~kg} / \mathrm{s}]$} & $\boldsymbol{\omega}_{2} \operatorname{diag}[10,10]$ & $\operatorname{diag}[2,2]$ \\
\hline$I_{x x}$ & 0.082 & {$\left[\mathrm{~kg} \cdot \mathrm{m}^{2}\right]$} & $K_{4}$ & 0.5 & {$[\mathrm{~kg} / \mathrm{rad}]$} & & $\operatorname{diag}[20,20]$ \\
\hline$I_{y y}$ & 0.082 & {$\left[\mathrm{~kg} \cdot \mathrm{m}^{2}\right]$} & $K_{5}$ & 0.5 & {$[\mathrm{~kg} / \mathrm{rad}]$} & & $\operatorname{diag}[20,20]$ \\
\hline$I_{z z}$ & 0.149 & {$\left[k g \cdot m^{2}\right]$} & $K_{6}$ & 0.5 & {$[\mathrm{~kg} / \mathrm{rad}]$} & & $\operatorname{diag}[3,3]$ \\
\hline
\end{tabular}

In order to verify the efficiency of the proposed method for quadrotor aircraft trace tracking in the presence of system parameter uncertainties and external disturbance, simulation is conducted using matlab. The system parameters, LESO parameters and controller parameters are listed in Table 1.

Table 1 The values of the quadrotor, LESO and Controller parameters 


$$
\left\{\begin{array}{l}
\boldsymbol{d}_{1}=0.4 \boldsymbol{g}_{2} \boldsymbol{x}_{3}+\left[\begin{array}{l}
-K_{1} \dot{x} / m+2 \\
-K_{2} \dot{y} / m+2
\end{array}\right] \\
\boldsymbol{d}_{2}=0.4 \boldsymbol{g}_{4} \boldsymbol{U}_{1}+\left[\begin{array}{l}
-K_{5} l \dot{\theta} / m+2 \sin (2 \pi t / 10) \\
-K_{4} l \dot{\phi} / m+2 \sin (2 \pi t / 10)
\end{array}\right] \\
\boldsymbol{d}_{3}=0.4 \boldsymbol{g}_{6} \boldsymbol{U}_{2}+\left[\begin{array}{c}
-K_{3} \dot{z} / m+2 \\
-K_{6} l \dot{\psi} / m+2 \sin (2 \pi t / 10)
\end{array}\right]
\end{array}\right.
$$

A spiral curve is chosen as the desired reference trace, since in the tasks like 3D structural scanning, quadrotor is required to move along a spiral curve. The spiral curve is defined as $x(t)=\sin (t / 10) m, y(t)=\cos (t / 10) m, z(t)=0.1 t m, \psi(t)=0.5 \sin (t / 10) \mathrm{rad}$. The total disturbance consisting of parameter uncertainties and external wind force is shown in Eq. 40. To further demonstrate the efficiency of the proposed LESO based backstepping method (BLF w LESO), it is compared with barrier Lyapunov function based backstepping control without LESO (BLF w/o LESO) and control Lyapunov function based backstepping control without LESO (CLF w/o LESO).

The trace tracking error constraints are set as $\boldsymbol{k}_{b 1}=\operatorname{diag}[0.08,0.08], \boldsymbol{k}_{b 2}=\operatorname{diag}[0.08,0.08]$. The trace tracking errors under the three methods mentioned above are shown from Fig.2 to Fig.5. Solid line shows the trace tracking error under (BLF w LESO) method, dash-dot line indicates the trace tracking error under (BLF w/o LESO) method and dot line is the trace tracking error under (CLF w/o LESO) method.

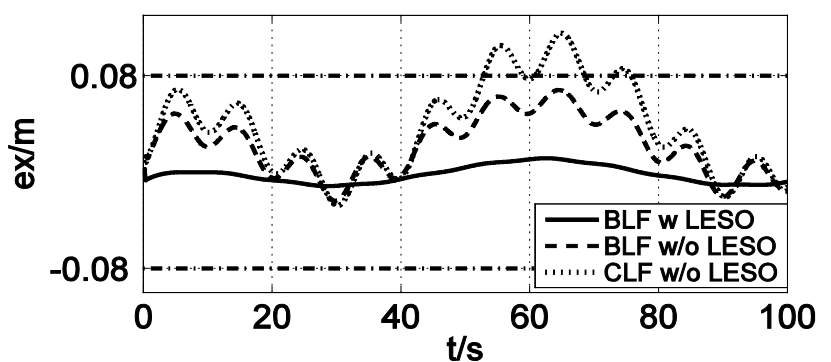

Fig. 2 Trace tracking error $e x(t)$

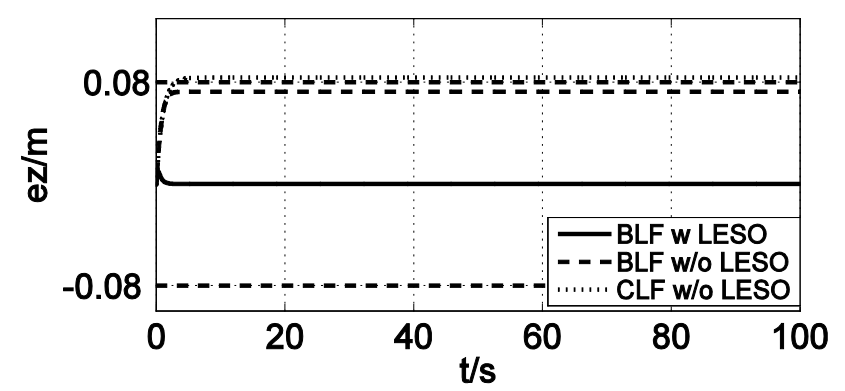

Fig. 4 Trace tracking error $e z(t)$

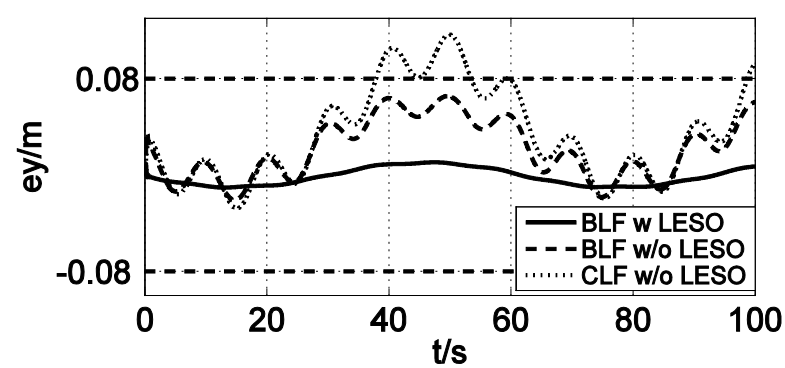

Fig. 3 Trace tracking error $e y(t)$

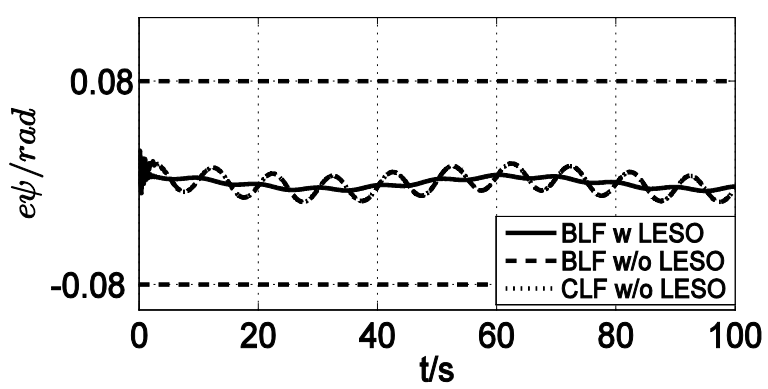

Fig. 5 Trace tracking error $e \psi(t)$

As seen from simulation results, $e x(t), e y(t), e \psi(t)$ under the three methods are all shown periodic fluctuation due to periodicity of the reference signal. The effect of rapid varying disturbance is clearly seen under (BLF w/o LESO) and (CLF w/o LESO) methods, because those two methods are lack of the capacity to estimate and compensate disturbance. (BLF w LESO) method shows the best performance due to its capacity to estimate and compensate disturbance, and trace tracking error under (BLF w LESO) method all satisfy trace tracking error constraints. In Fig.2, $e x(t)$ under (BLF w LESO) method is not only within the tolerance $0.08 \mathrm{~m}$ but smaller than $0.015 \mathrm{~m}$. Compared with $e x(t)$ under (BLF w LESO) method, $e x(t)$ is much larger under (BLF 
w/o LESO) method. (CLF w/o LESO) method shows the worst performance, $e x(t)$ under (CLF w/o LESO) method violates error constraint and surpass $0.1 \mathrm{~m}$. Fig. 3 shows the similar results as in Fig.2. As seen from Fig.4, $e z(t)$ under (BLF w LESO) method is close to zero. Fig.5 shows the trace tracking error $c \psi(t)$ of the three methods, $c \psi(t)$ are all satisfied the error constraints. (BLF w LESO) method shows the best performance with $c \psi(t)$ smaller than 0.006 rad.

The error and energy comparison of the three methods is shown in Table 2. It can be seen from Table 2 that ISE of (BLF w LESO) method is much smaller than the other two methods which shows the accuracy of the proposed method. In the view of energy consumption, there is not much difference between those three methods, (CLF w/o LESO) shows little higher energy consumption than the other two methods.

Table 2 Error and Energy Comparison

\begin{tabular}{c|c|c}
\hline \hline Method & ISE & Energy Comsumption \\
\hline BLF w LESO & 0.02 & 50.74 \\
BLF w/o LESO & 0.13 & 50.46 \\
CLF w/o LESO & 0.17 & 55.12 \\
\hline \hline
\end{tabular}

\section{Conclusion}

In this paper, LESO based backstepping method using barrier Lyapunov function is proposed for quadrotor trace tracking with output tracking error constraints. LESO is designed to estimate quadrotor uncertainties which consist of model parameters variation and external wind disturbance. Based on the estimated uncertainties, backstepping method using barrier Lyapunov function is constructed to attenuate the effect of system uncertainties and ensure the tracking error remain within boundary. Simulation results show the efficiency of the proposed method. Trace tracking error constraints are satisfied via backstepping method using barrier Lyapunov function. And LESO helps improve the performance of quadrotor trace tracking.

\section{Reference}

[1] F. Yacef, O. Bouhali, M. Hamerlain, “Adaptive fuzzy tracking control of unmanned quadrotor via backstepping”, Proc. IEEE 23rd International Symposium on Industrial Electronics. HangZhou, China, May 28-31, 2014, pp.40-45.

[2] S. Bouabdallah, A. Noth, and R. Siegwart, "PID vs LQ control techniques applied to an indoor micro quadrotor”, Proc. Intelligent Robots and Systems. Senda, Japan, Sep 28-Oct 2, 2004, pp. 2451-2456.

[3] K. Alexis, G. Nikolakopoulos, A. Tzes. "Model predictive control scheme for the autonomous flight of an unmanned quadrotor", Proc. IEEE 20rd International Symposium on Industrial Electronics. Gadansk, Poland, Jun 27-30, 2011, pp. 2243-2248.

[4] Jingjing. X, and Enhui. Z, "Position and attitude tracking control for a quadrotor UAV", Isa Transactions. vol. 52, no. 3, 2014, pp. 725-731.

[5] T. Madani and A. Benallegue, "Backstepping Control for a Quadrotor Helicopter", Proc. IEEE/RSJ International Conference on Intelligent Robots and Systems. Beijing, China, Oct 9-15, pp. 3255 - 3260.

[6] Jinqing. Han, “Active disturbance rejection controller and its applications”, Control Decis. vol. 13, no. 1, 1998, pp. 19-23. 
[7] Lu. Wang, Jianbo. Su, “Attitude tracking of aircraft based on disturbance rejection control”, Control Theory and Application. vol. 30, no. 12, 2013, pp.1608-1615. [in chinese].

[8] Wenchao. Xue, Yi. Huang, “On performance analysis of ADRC for nonlinear uncertain systems with unknown dynamics and discontinuous disturbances”, Proc. 32nd Chinese Control Conference. Xi’an, China, July 26-28, 2013, pp.1102-1107.

[9] S. Bouabdallah, R. Siegwart, "Full Control of a Quadrotor", Proc IEEE/RSJ International Conference on Intelligent Robots and Systems. San Diego, CA, USA, Oct 29 - Nov 2, 2007, pp.153-158

[10] Xiaoxia. Y and Yi. H, “Capabilities of extended state observer for estimating uncertainties”, Proc. 2009 American Control Conference, St. Louis, MO, USA, June 10-12, 2009, pp. 3700 - 3705. 